\title{
RNA under Tension: Folding Landscapes, Kinetic Partitioning Mechanism, and Molecular Tensegrity
}

\author{
Jong-Chin Lin, ${ }^{\dagger}$ Changbong Hyeon, ${ }^{\ddagger}$ and D. Thirumalai* ${ }^{\dagger}$ \\ ${ }^{\dagger}$ Biophysics Program, Institute for Physical Science and Technology, University of Maryland, College Park, Maryland 20742, United \\ States \\ ${ }^{\ddagger}$ School of Computational Sciences, Korea Institute for Advanced Study, Seoul 130-722, Republic of Korea
}

ABSTRACT: Noncoding RNA sequences play a great role in controlling a number of cellular functions, thus raising the need to understand their complex conformational dynamics in quantitative detail. In this Perspective, we first show that single-molecule pulling experiments when combined with theory and simulations can be used to quantitatively explore the folding landscape of nucleic acid hairpins, and riboswitches with tertiary interactions. Applications to riboswitches, which are noncoding RNA elements that control gene expression by undergoing dynamical conformational changes in response to binding of metabolites, lead to an organization principle in which assembly of RNA is determined by the stability of isolated helices. We also point out the limitations of single-molecule pulling experiments, with molecular extension as the only accessible parameter, in extracting key parameters of the folding landscapes of RNA molecules.

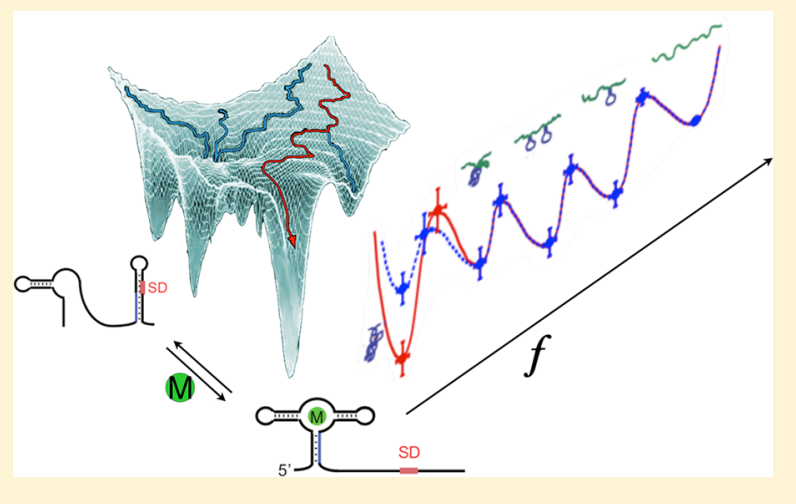

$\mathrm{E}$ ver since the pioneering discovery that RNA molecules can act as enzymes, an increasing repertoire of functions have been associated with these bewilderingly complex biological molecules. ${ }^{1}$ Even the ribosome that helps in protein synthesis can be legitimately considered to be a ribozyme (RNA enzyme) because the peptidyl transfer center, the site at which the peptide bond is formed, is devoid of proteins. ${ }^{2}$ More recently, it has been discovered that RNA interference, involving small interference RNA and microRNA, plays a vital role in post transcriptional gene regulation. ${ }^{3}$ The finding that riboswitches, ${ }^{4,5}$ a class of RNA molecules, can by themselves control gene expression, without the participation of proteins, further underscores the important role RNA plays in key cellular functions. In a majority of these examples, folding and conformational changes associated with RNA molecules are at the center stage.

\section{The recognition that RNA plays a vital role in many biological functions has greatly elevated the need to quantitatively under- stand their folding using a variety of tools.}

RNA folding is considerably more complicated than the better studied protein folding for a number of reasons. ${ }^{6}$ First, the building blocks of RNA (A, U, G, and C) are chemically similar except for the size and shapes of the bases. As a consequence, RNA molecules are closer to homopolymers than proteins. Second, only about $50 \%$ of nucleotides in RNA form Watson-Crick base pairs (A-U and G-C), whereas the remaining nucleotides are in bulges, loops, and other architectures. ${ }^{7}$ Finally, the highly charged nature of the phosphate groups, which makes RNA a polyelectrolyte, implies that folding to a compact structure cannot take place without the presence of counterions. ${ }^{8}$ Some of these aspects are reflected in the stability gap (the difference in the free energies of the native and low-lying structures) for RNA being not as large as it is in proteins. ${ }^{6,9}$ Thus, during the folding process, RNA can readily adopt alternate folds, which, while making the extraordinary range of functions that are associated with RNA possible, also makes the study of their folding complicated.

The complexity of RNA folding is succinctly summarized using the kinetic partitioning mechanism (KPM) according to which a pool of unfolded molecules partitions into two distinct populations $^{10}$ that reach the native basin of attraction (NBA) by vastly different time scales under folding conditions. A fraction, $\Phi$ of unfolded molecules, folds rapidly to the NBA while the remaining fraction $(1-\Phi)$ is kinetically trapped in multiple competing basins of attraction (CBAs) (Figure 1a). Transitions from the CBAs to the NBA, which occur by partial or global unfolding of the conformations in the CBAs, ${ }^{11}$ could take minutes or longer as shown experimentally for the well studied Tetrahymena ribozyme with $\Phi \approx 0.1 .^{11,12}$ More

Received: September 28, 2012

Accepted: November 19, 2012 

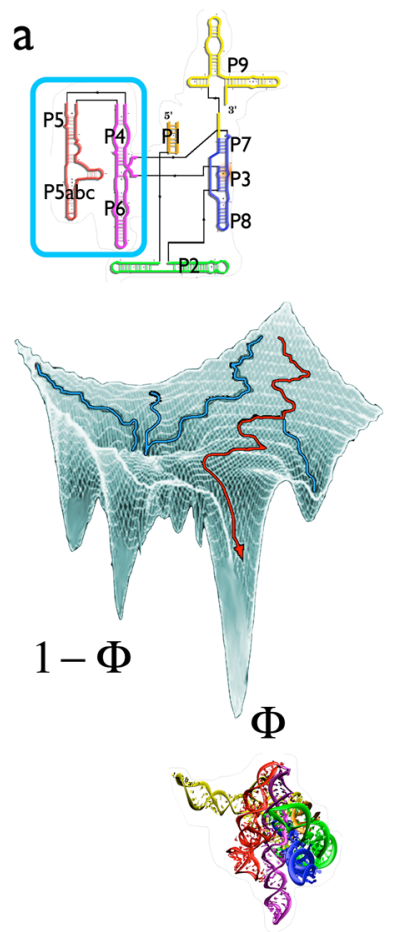

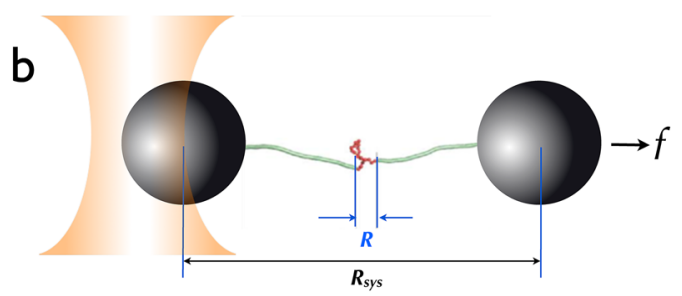

C

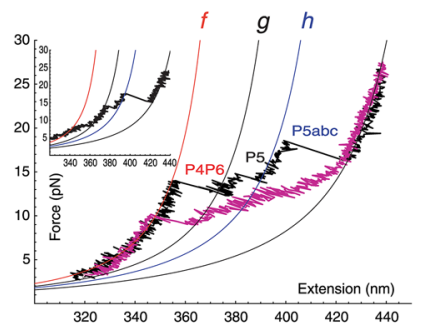

d

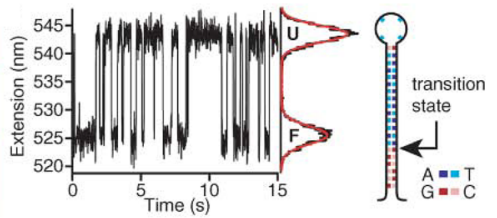

Figure 1. (a) A schematic of folding landscape and kinetic partitioning. Secondary structure map and folded states of Tetrahymena ribozyme are depicted on the top and bottom of the landscape cartoon. The P4-P6 domain is highlighted in the cyan box. (b) Illustration of the setup of a LOT experiment for a RNA hairpin. The figure illustrates that the end-to-end distance $(R)$ dynamics of RNA hairpin is indirectly monitored through the dynamics of distance between the two microbeads $\left(R_{\text {sys }}\right)$. (c) FEC of the P4-P6 domain of Tetrahymena ribozyme. Order of unfolding of the structural elements in the P4-P6 domain are extracted from the measured FECs as indicated. (d) Histogram of end-to-end distance (extension) $(P(R))$ of a DNA hairpin (shown on the right) are obtained directly from the time trace of $R_{\text {sys }}(t)$. The graphics in panels $\mathrm{c}$ and $\mathrm{d}$ are adapted with permission from refs 16 and 17. Copyright 2003 and 2006 American Association for the Advancement of Science.

recently, temperature jump experiments of RNA pseudoknots ${ }^{13}$ show that the KPM quantitatively describes their folding.

Although global folding of RNA molecules is well understood, the details of the underlying folding landscape are only starting to emerge thanks in part to advances in single-molecule pulling laser optical tweezers (LOT) experiments. ${ }^{14}$ In LOT experiments, the ends of RNA of interest are tethered to handles (DNA or hybrid DNA/RNA), which are themselves attached to spherical beads that are localized by laser traps (Figure 1b). Mechanical force can be transmitted to RNA by moving one of the beads at a constant loading rate, $r_{\mathrm{f}}=k_{\text {trap }} v$, ( $k_{\text {trap }}$ is the force constant associated with the harmonic trap potential, and $v$ is the pulling velocity). In this mode, the experiments yield force as a function of extension, $R$, of the RNA (Figure 1c) referred to as force-extension curves (FECs). ${ }^{15,16}$ The FECs could be used to infer the order in which the structural elements are ruptured as the force is increased. ${ }^{16}$ Alternatively, constant $f$, applied to the ends of RNA by suitable feedback techniques, could be used to generate mechanical folding trajectories expressed, which yield, $R_{\text {sys }}(t)$ (Figure $1 \mathrm{~b}$ ) projected along the force axis as a function of time, $t$ (Figure 1d provides a sample trajectory for a DNA hairpin $\left.{ }^{17}\right)$. In analyzing the $R_{\text {sys }}(t)$ data to obtain the equilibrium free energy profile $F(R)$ as a function of $R$ (Figure $1 \mathrm{~b}$ for the difference between $R$ and $R_{\text {sys }}$ ), a few assumptions are made: (i) Transverse fluctuations at the applied forces are negligible. (ii) Dynamics of $R_{\text {sys }}(t)$ mirrors $R(t)$. (iii) The system of interest ergodically samples the conformational space on the observation time scale so that the extracted $F(R)$ from $R(t)$ by appropriate deconvolution methods are the equilibrium profiles. Despite the restriction that LOT experiments only provide one-dimensional $F(R)$, they have given insights into folding of RNA at the single-molecule level. In this Perspective, we show that simulations based on coarse-grained models ${ }^{18,19}$ of RNA hairpin and riboswitches, experimental data, and theoretical considerations can be combined to extract some key aspects of the folding landscapes and dynamics. Our purpose here is not to merely compare theoretical predictions and experiments, but rather show both the successes and ultimately the challenges that need to be overcome in order to realize the potential of the ideas, methods, and concepts sketched here.

\section{Remarkably, formation of a hair- pin, the simplest structural motif, is complex and very much de- pends on the specific region of the landscape from which folding commences.}

Complexity of RNA Hairpin Formation. RNA hairpins are the simplest but the most ubiquitous motifs that form the building blocks of higher order structures. A substantial energetic contribution to the folded states of RNA comes from base pairings, the majority of which participate in forming stem-loop structures, namely hairpins. For over four decades, considerable effort has been made to study the thermodynamics and kinetics of the folding of simple RNA hairpins by using ensemble measurements, initiated by temperature $(T)$ jump. ${ }^{20-24}$ These 

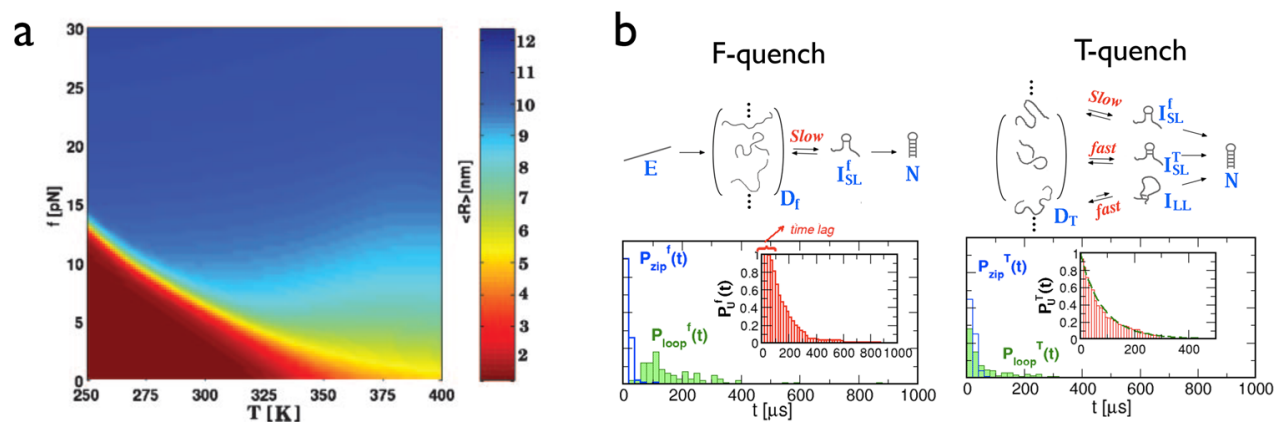

Figure 2. (a) $(T, f)$ phase diagram of a P5GA hairpin using $R$ (scale on the right) as an order parameter. Blue is unfolded, and dark red corresponds to the hairpin state. (b) Demonstration that the folding mechanisms of a P5GA hairpin vary depending on the protocol used to initiate folding. On the left we show the schematics of approach to the hairpin state using force-quench, and the right shows the events upon temperature-quench. The bottom graphs show decompositions of folding time $\left(\tau_{\mathrm{FP}}\right)$ under these protocols into times for looping $\left(\tau_{\text {loop }}\right)$ and zipping time $\left(\tau_{\text {zip }}\right)$. While the distributions of $\tau_{\text {zip }}$ are similar for both conditions, the $\tau_{\text {loop }}$ upon $f$-quench is longer than under $T$-quench conditions and is more broadly distributed. The fraction of unfolded molecules at each condition $\left(P_{\mathrm{U}}^{f}(t)\right.$ and $\left.P_{\mathrm{U}}^{T}(t)\right)$ is plotted in the inset. $P_{\mathrm{U}}^{f}(t)$ that is fit to $P_{\mathrm{U}}^{f}(t)=(t)=\mathrm{e}^{-(t-50 \mu \mathrm{s}) / 138 \mu \mathrm{s}}$ for $t>$ $50 \mu \mathrm{s}$ shows a lag phase at $0<t<50 \mu \mathrm{s}$, suggesting that the state $I_{\mathrm{SL}}^{f}$ is an obligatory step for the refolding process under $f$-quench. By contrast, $P_{\mathrm{U}}^{T}(t)$ is well fit using a sum of two exponential functions $P_{\mathrm{U}}^{T}(t)=0.44 \times \mathrm{e}^{-t / 63 \mu \mathrm{s}}+0.56 \times \mathrm{e}^{-t / 104 \mu \mathrm{s}}$.

experiments showed that, generically, RNA hairpins fold in an approximate two-state manner with a time constant of about 10 $\mu$ s. Recent high-resolution ensemble experiments ${ }^{25,26}$ and single-molecule pulling experiments ${ }^{15,27}$ have shown that hairpin formation could be more complex than previously thought. Single-molecule force measurements of RNA hairpin dynamics using LOT, pioneered by Liphardt et al., ${ }^{15}$ showed that a single RNA hairpin undergoes reversible folding and unfolding transitions in a narrow range of $f$ values $(13-15 \mathrm{pN})$. The trajectory measuring the time-dependent changes in the extension, $R(t)$, jumps predominantly between two values: one corresponding to the folded state, and the other to the unfolded state. To a first approximation, such folding trajectories could be analyzed using an apparent two-state model, just as in the classic $T$-jump experiments.

A long single time trace of RNA hairpin generated in LOT experiments is sufficient to extract both the thermodynamic and kinetic features of hairpin dynamics as long as it is assured that the RNA molecule ergodically samples its conformational space. $^{15,28}$ By using the average dwell times of RNA in the folded (small values of $R(t)$ ) and unfolded states (large values of $R(t)$ ) as a function of $f$, the equilibrium constant between the two states, $K_{\text {eq }}(f)=\tau_{\mathrm{U}}(f) / \tau_{\mathrm{F}}(f)$, has been directly measured. Extrapolation of the measurements to $f=0$ showed that the extracted stability values of P5ab and P5abc, the core secondary elements of the P4-P6 domain of the Tetrahymena ribozyme, are consistent with those from bulk measurement $\left(K_{\mathrm{eq}}(f \rightarrow 0)\right.$ $\left.\approx K_{\mathrm{eq}}^{\mathrm{o}}\right),{ }^{15}$ thus reinforcing the two state model for folding thermodynamics of generic RNA hairpins. A more detailed view of RNA hairpin thermodynamics was provided using realistic simulations, ${ }^{29}$ which obtained the equilibrium phase diagram of a 22-nt RNA hairpin as a function of $f$ and $T$ (Figure $2 \mathrm{a})$. The phase diagram also revealed that there are predominantly only two states, although this study provided hints of fine structure in the phase diagram. The two states are separated by a first-order phase coexistence line expressed in terms of a set of critical points $\left.\left(T_{\mathrm{m}}, f_{\mathrm{m}}\right)\right)^{29,30}$ Thus, from a thermodynamic perspective, it appears that the phase diagram of a RNA hairpin in the $T$ and $f$ variables could be approximated as a two-state system.

Where Folding Starts Matters. The two-state model for RNA hairpins hides the complex dynamics of the RNA hairpin, which have been recently revealed using realistic simulations by varying $f$ and $T,{ }^{30}$ and by advances in experimental methods. ${ }^{31}$ In addition, recent $T$-jump kinetic experiments showed that hairpins form in multiple steps, ${ }^{25,26}$ which challenges the conventional notion that small nucleic acid hairpins could be modeled using only two states. The structural origin of the complexity is due to the link between the establishment of local base-pairs and the global hairpin formation, which were clearly demonstrated using coarse-grained simulations ${ }^{29,32}$ and detailed all-atom models. ${ }^{33,34}$ Hyeon and Thirumalai showed that the folding landscape hairpin formation requires at least two reaction coordinates. ${ }^{35,30}$ Besides $R$, a collective variable describing the average deviation of loop dihedral angles from the native value is needed. Two-dimensional free energy surface at $f=f_{\mathrm{m}} \approx 0$ and $T=T_{\mathrm{m}}$, calculated using these variables shows that the folding landscape of even a simple RNA hairpin is rugged, (especially at high $T_{\mathrm{m}}$ and low $f_{\mathrm{m}}$ ), explaining the observed complex kinetics. 25,26

The complexity of the kinetics of RNA hairpin formation is evident when folding is initiated from different parts of the landscape, which can be achieved by preparing the initial conformations by $T$-jump or by using high stretching forces. Remarkably, the refolding pathways of hairpin formation from a fully stretched initial state upon $f$-quench are distinct from the folding pathways observed in $T$-quench refolding (Figure $2 b$ ). The initial conformations of RNA hairpin under high tension are fully stretched and are structurally homogeneous. The various conformations largely differ in the internal degrees of freedom while the overall end-to-end distance is large, resulting in substantial deviations of the conformation of the tetra loop from the native structure. Thus, the first step in the hairpin formation from the initially stretched conformations is the tetra-loop formation, corresponding to the slow nucleation $\left(I_{\mathrm{SL}}^{f}\right.$ state in Figure $2 b$ ) stage. The high entropic cost to establish the correct loop dihedral angles makes the loop formation dynamics unusually slow. Subsequent to the nucleation step, the zipping of remaining base pairs leads to rapid hairpin formation. Thus, the hairpin forms by the classic mechanism (establishment of base pair contact near the loop followed by a zipping process) when folding is initiated by $f$-quench (Figure $2 b)$.

By contrast, upon $T$-quench, refolding commences from a broad thermal ensemble of unfolded conformations (Figure $2 b$ ). As a result, nucleation can originate from regions other 
a

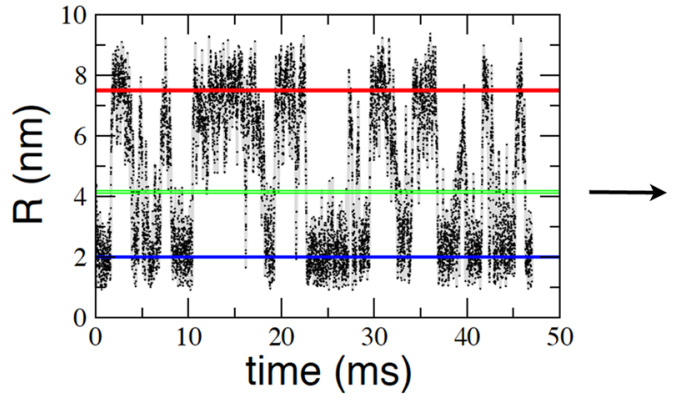

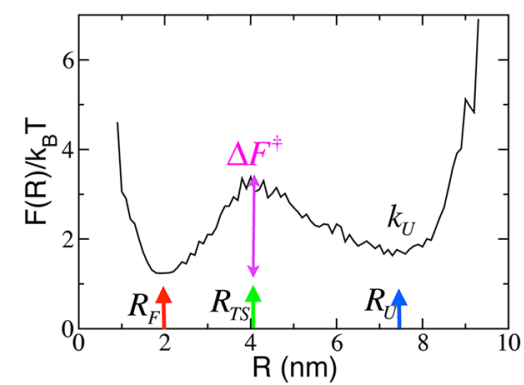

b

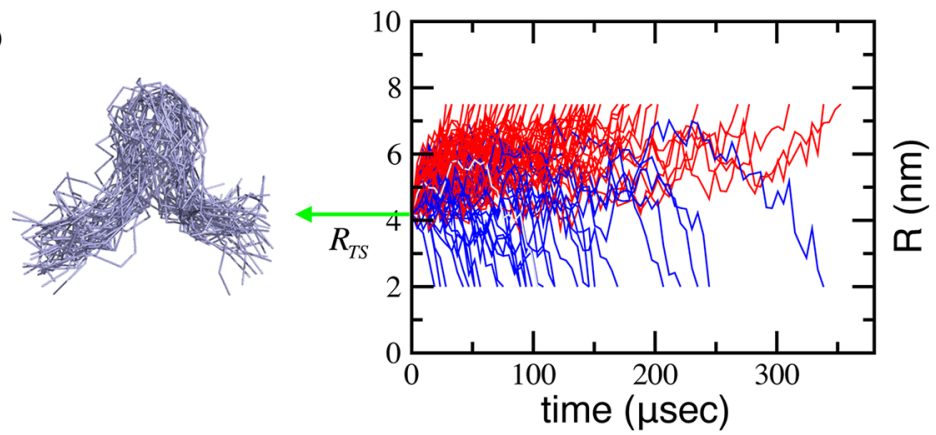

Figure 3. (a) Time trace of end-to-end distance $(R)$ of RNA hairpin at transition midforce $f_{\mathrm{m}}=14.7 \mathrm{pN}$ (left). The corresponding free energy profile in terms of $R, F(R)$ (right). The positions of native, unfolded, and transition states are marked with arrows. In addition, barrier height $\left(\Delta F^{*}\right)$ and the curvature of the unfolded state $\left(k_{\mathrm{U}}\right)$ are also shown on the $F(R)$. (b) Time trajectories of simulations starting from the configurations of the transition state ensemble (shown on the left). Trajectories reaching the folded and unfolded state at 2.0 and $7.5 \mathrm{~nm}$ are colored in blue and red, respectively. In the blue trajectories, a number of recrossing events can be observed. The figures are adapted with permission from refs 29 (Copyright 2005 National Academy of Sciences) and 30 (Copyright 2008 American Chemical Society).

than near the tetra-loop $\left(f_{\mathrm{SL}}^{f}, I_{\mathrm{SL}}^{T}\right.$, and $I_{\mathrm{LL}}$ states in Figure $2 \mathrm{~b}$ ). Consequently, the pathway diversity is greater when hairpin formation is initiated by $T$-quench rather than $f$-quench. The differences in the folding mechanism between these two methods to trigger hairpin formation are entirely due to the variations in the initial conformations. Exploring the details of the heterogeneous kinetics requires multiple probes that control the conformations of the ensemble of unfolded states. Our studies also showed that the complexity of energy landscape observed in ribozyme experiments is already reflected in the formation of simple RNA hairpins.

Folding Landscapes from Pulling Experiments. Besides yielding stability and hopping rates between various states, singlemolecule pulling experiments have been used to obtain onedimensional free energy profiles as a function of $R$. It should be noted that the only directly measurable quantities in LOT experiments are the time-dependent changes in the distance between the beads, $R_{\text {sys }}(t)$ (Figure 1b), at a fixed $f$. What is of interest, however, is the free energy profile, $F(R)$ as a function of $R$. The complicated problem of going from $R_{\text {sys }}(t)$ to $P(R)$, the probability that the extension is between $R$ and $R+d R$ by accounting for fluctuations of the semiflexible polymer handles and bead motions, has been solved using a number of ad hoc ${ }^{27}$ and precise theoretical methods. ${ }^{36}$ Assuming that $P(R)$ can be extracted from $R_{\text {sys }}(t)$, the free energy profile can be computed using $F(R)=-k_{\mathrm{B}} T \ln P(R)$. For approximate two-state systems, as is the case in P5GA hairpin ${ }^{28,37}$ or DNA hairpins, ${ }^{14,17} F(R)$ has two dominant minima separated by a single barrier located at $R=R_{\mathrm{TS}}$ (Figure 3). At the transition midforce $f=f_{\mathrm{m}}$, the probability of residing in the two basins of attraction should be identical (Figure 3a), implying $\int_{0}^{R_{\mathrm{TS}}} \mathrm{e}^{-\beta F_{\mathrm{m}}(R)} \mathrm{d} R=$ $\int_{R_{\mathrm{TS}}}^{\infty} \mathrm{e}^{-\beta F_{\mathrm{m}}(R)} \mathrm{d} R$. Alternatively, $f_{\mathrm{m}}$ can also be measured by equating the average dwell times in the NBA and unfolded basin of attraction (UBA) $\tau_{\mathrm{F}}=\tau_{\mathrm{U} \cdot}{ }^{15,38,39}$ Accurate $F(R)$ profiles give estimates of the free energy barrier, $\Delta F^{\ddagger}$ and $R_{\mathrm{TS}}$, both of which are functions of $f$. The accuracy of these estimates depends on the assumption that $R$ is a good reaction coordinate, and that no information is lost in converting the measured folding trajectories $\left(R_{\text {sys }}(t)\right.$ as a function of $\left.t\right)$ to $F(R)$.

There are two limitations that prevent extraction of the complete shape of $F(R)$. First, the probability $P\left(R_{T S}\right)$ of reaching the transition state is small, making it difficult to obtain data in the neighborhood of $R_{\mathrm{TS}}$. Second, the inferred profiles hide the possibility that there is roughness (on the length scale corresponding to base pair rupture) superimposed on the smooth $F(R)$. These limitations were recently overcome in ingenious experiments on DNA hairpins by La Porta and coworkers, ${ }^{31}$ who used a harmonic constraint to restrict $R$ to arbitrary values for long enough times to collect excellent statistics so that reliable estimates of $P(R)$ could be made (Figure 4a). This method, which is an experimental realization of the popular umbrella sampling used in computer simulations to obtain potentials of mean force, revealed fine structure in $F(R)$ for DNA hairpins. The superimposed fine structure on previously inferred smooth profiles perhaps reflects the rupture of base pairs (Figure 4a), which manifests itself as "roughness" in the folding landscape. More importantly, this study showed that $R_{\mathrm{TS}}$ and the width of the transition region (see below on the potential relevance of $R_{\mathrm{TS}}$ ) at a given $f$ can be directly inferred from measurement. It would be of great interest to apply this unique experimental method to study RNA molecules with tertiary interactions.

Hopping Rates and Free Energy Profiles. What is the utility of $F(R)$ for biomolecules if the goal is to obtain the thermodynamics and kinetics at zero or low forces in the 
a

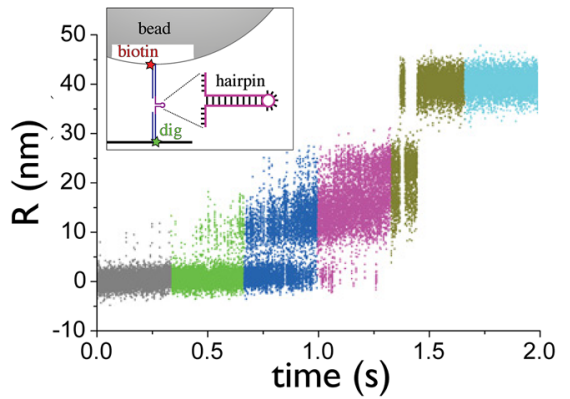

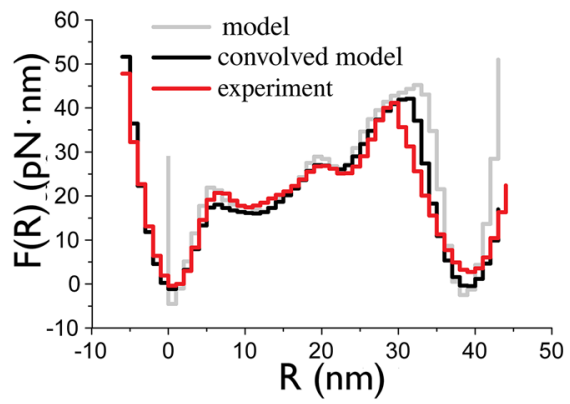

b
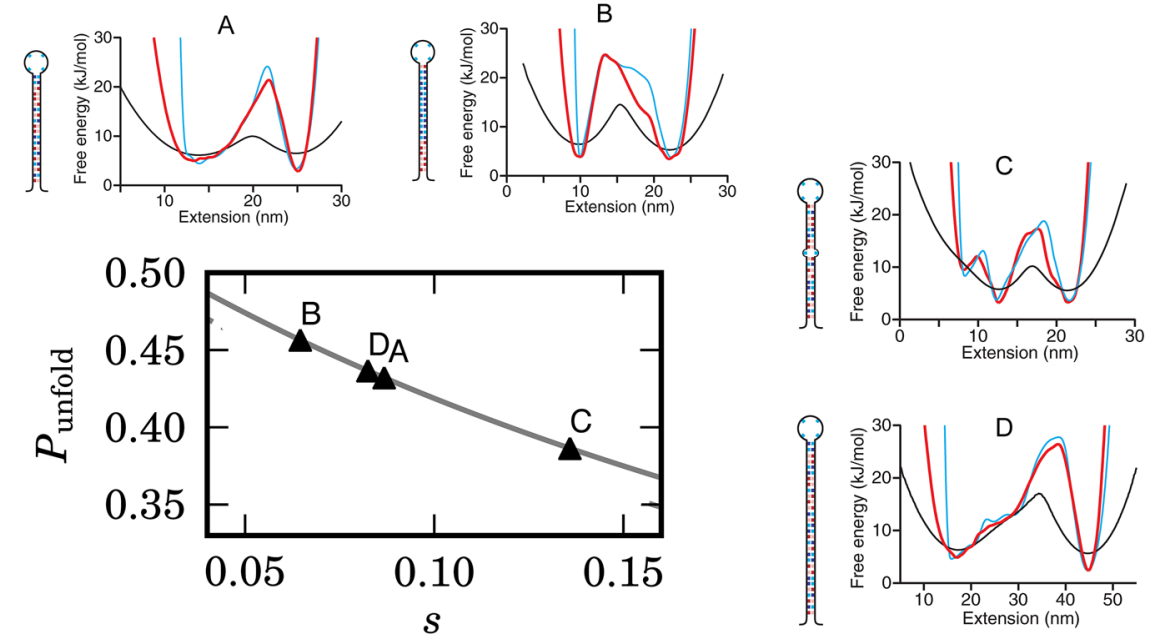

Figure 4. (a) End-to-end distance dynamics of DNA hairpin under gradually increasing harmonic constraint. Gradual change of R-dynamics is depicted in the folding trajectory trajectory. Free energy profile reconstructed by using the harmonic constraining method (umbrella sampling) at the transition midforce is shown on the right. (b) Tensegrity parameters calculated for four DNA hairpins with different sequences in ref 17 is related to $P_{\text {unfold }}$. The DNA hairpin with sequence B is predicted to have $P_{\text {unfold }}$ most proximal to 0.5 , which suggests that the free energy profile calculated in terms of end-to-end distance coordinate most accurately describes the dynamics of this DNA hairpin. The figure is adapted with permission from refs 17 (Copyright 2006 American Association for the Advancement of Science) and 45 (copyright 2011 American Physical Society).

absence of handles and beads? Although this question has not been fully answered, several groups routinely use the measured $F(R)$ and the hopping kinetics (between the folded and unfolded states in the case of a hairpin) at finite $f$ to extract rates at $f=0$ as well as the associated barrier heights $\left(\Delta F^{\ddagger}\right)$ by assuming that $R$ is an excellent reaction coordinate. ${ }^{17,40}$ Because independent measurements of the absolute values of the $\Delta F^{\ddagger}$ are difficult to make, the reliability of the extracted values cannot be easily assessed. Two computational studies, using RNA hairpin and riboswitches as illustrations, have shown the potential utility of one-dimensional folding landscapes in obtaining accurate rates over a narrow range of forces close to $f_{\mathrm{m}}{ }^{37,41}$ In these examples, the intrinsic rates can be independently calculated using the trajectories generated in the full dimensional landscape, thus allowing for a quantitative comparison with results obtained from the projected $F(R)$. It is now firmly established that accurate $F(R)$ can be obtained by attaching handles that are stiff, ${ }^{17,28,35}$ implying that the ratio $L /$ $l_{\mathrm{p}}\left(L\right.$ and $l_{\mathrm{p}}$ are the contour length and the persistence length of the handles, respectively) should be as small as possible. If $F(R, f)$ at $f=f_{\mathrm{m}}$ is known accurately, then the profiles at arbitrary values of $f$ may be obtained using the Zhurkov-Bell relation ${ }^{42,43} F(R, f)=F\left(R, f_{\mathrm{m}}\right)-\left(f-f_{\mathrm{m}}\right) R$. If $R$ is a good reaction coordinate (all other coordinates have equilibrated on time scales less than the hopping times so that the slow dynamics occurs on $F(R)$ ), then the hairpin formation time can be calculated using standard mean first passage time formalism,

$$
k(f)^{-1}=\frac{1}{D_{\mathrm{U} \rightarrow \mathrm{F}}} \int_{R_{\mathrm{F}}}^{R_{\mathrm{U}}} \mathrm{d} x \mathrm{e}^{\beta F(x)} \int_{x}^{\infty} \mathrm{d} y \mathrm{e}^{-\beta F(y)}
$$

provided that the diffusion coefficient for transition from $\mathrm{U} \rightarrow$ $\mathrm{F}$ is known. For the P5GA hairpin, we showed that this method gives reliable results for hopping rates over a range of $f$ around $f_{\mathrm{m}}$ provided that the diffusion coefficient is calibrated by equating the theoretically calculated time at $f_{\mathrm{m}}$ to the simulated value. ${ }^{28}$ It might be tempting to use our method for obtaining rates at $f=0$, but this would not be justified a priori.

Molecular Tensegrity and the Transition State. Another parameter that is extracted from $F(R)$ or suitable fits to $f$ dependent hopping rates is the location of the transition state, $R_{\mathrm{TS}}$, which in principle moves as $f$ changes. ${ }^{35}$ For $R_{\mathrm{TS}}$, associated with the barrier top of $F(R)$ at $f=f_{\mathrm{m}}$, to be considered the "true" transition state, it is necessary to ensure that it is consistent with other conventional definitions of the transition state ensemble. A plausible definition of the TS is that the forward (to the unfolded state, $P_{\text {unfold }}$ ) and backward (to the folded state, $P_{\text {fold }}$ ) fluxes starting from the transition state on the reaction coordinate should be identical. ${ }^{44}$ For the hairpin it means that if an ensemble of structures were created starting at $R_{\mathrm{TS}}$, then the dynamics in the full multidimensional space would result in these structures reaching the folded and unfolded states with equal probability. The number of events reaching $R_{\mathrm{F}}$ and $R_{\mathrm{U}}$ starting from $R_{\mathrm{TS}}$ can be directly counted if folding trajectories with high temporal resolution exhibiting multiple folding and unfolding transitions at $f=f_{m}$ can be 
generated (Figure 3a). Our coarse-grained simulations, which are the first to assess the goodness of $R_{T S}$ as a descriptor of the TS, showed that, starting from $R_{\mathrm{TS}}$, the hairpin crosses the TS region multiple times before reaching $R=R_{\mathrm{F}}$ or $R_{\mathrm{U}}$, suggesting that the TS region is broad and heterogeneous. The transition dynamics of biopolymers occurs on a bumpy folding landscape with fine structure even in the TS region, which implies there is an internal coordinate determining the fate of trajectory projected onto the $R$-coordinate. In accord with this inference, we showed that for the P5GA hairpin the TS structural ensemble is heterogeneous (Figure $3 \mathrm{~b}$ ). More pertinently, the forward and backward fluxes starting from the structure in the TS ensemble (see the dynamics of trajectories starting from $R_{\mathrm{TS}}$ in Figure $3 \mathrm{~b}$ ) do not satisfy the equal flux condition, $P_{\text {fold }}=$ $P_{\text {unfold }}=0.5$. Thus, from a strict perspective, $R$ for a simple hairpin may not be a good reaction coordinate, even under tension, implying that $R$ is unlikely to be an appropriate reaction coordinate at $f \approx 0$.

\section{Simulations using coarse-grained models, which are remarkably successful in computing folding landscapes of riboswitches, and theoretical concepts such as mo- lecular tensegrity can be used to assess whether extension is a good reaction coordinate in sin- gle-molecule pulling experiments.}

On the basis of simulations, Morrison et al. ${ }^{45}$ proposed a fairly general theoretical criterion to determine whether $R$ could be a suitable reaction coordinate. The theory uses the concept of tensegrity (tensional integrity), which was introduced by Fuller and developed in the context of biology to describe the stability of networks. The notion of tensegrity has been used to account for cellular structures ${ }^{46}$ and more recently for the stability of globular proteins, ${ }^{47}$ the latter of which made an interesting estimate that the magnitude of inter-residue precompression and pretension, associated with structural integrity, can be as large as a few $100 \mathrm{pN}$. Using $F(R)$, the experimentally measurable molecular tensegrity parameter is defined as $s \equiv f_{\mathrm{c}} / f_{\mathrm{m}}=\Delta F^{\ddagger}\left(f_{\mathrm{m}}\right) / f_{\mathrm{m}} \Delta R^{\ddagger}\left(f_{\mathrm{m}}\right)$, where $\Delta F^{\ddagger}=$ $F\left(R_{\mathrm{TS}}\right)-F\left(R_{\mathrm{F}}\right)$ and $\Delta R^{\dagger}=R_{\mathrm{TS}}-R_{\mathrm{F}}$. The molecular tensegrity parameter $s$ represents a balance between the compression force $\left(f_{\mathrm{m}}\right)$ and the tensile force $\left(f_{\mathrm{c}}\right)$, a building principle in tensegrity systems. ${ }^{48}$ For hairpins, such stabilizing interactions are favorable base pair formations. In terms of $s$ and the parameters characterizing the one-dimensional landscape $\left(f_{\mathrm{m}}\right.$ and $k_{\mathrm{u}}$ in Figure $3 \mathrm{a}$ ), an analytic expression for $P_{\text {unfold }}$ has been obtained. $^{45}$ For $R$ to be a good reaction coordinate, it is required that $P_{\text {unfold }} \approx(1 / 2)$. The theory has been applied to hairpins and multistate proteins. Using experimentally determinable values for $s$, one can assess whether $R$ is a good reaction coordinate by calculating $P_{\text {unfold }}$ using theory. Applications of the theory to DNA hairpins ${ }^{45}$ show (Figure $4 \mathrm{~b}$ ) that the precise sequence determines whether $R$ can be reliably used as an appropriate reaction coordinate, thus establishing the usefulness of the molecular tensegrity parameter.
Riboswitches under Tension. Riboswitches are noncoding RNA elements that sense cellular signals and regulate gene expression by binding target metabolites. ${ }^{4,49}$ They contain a conserved aptamer domain, which can bind the metabolite, and a downstream expression platform that controls transcription termination or translation initiation (Figure 5a). Riboswitches

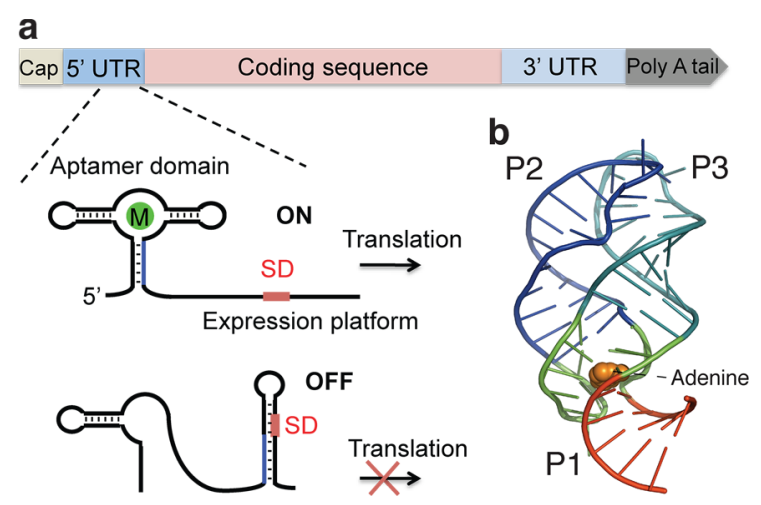

Figure 5. (a) Schematic of a riboswitch, which is in the $5^{\prime}$ untranslated region ( $5^{\prime} \mathrm{UTR}$ ) in mRNA. The add adenine riboswitch (blow up of the $5^{\prime}$ UTR given below) contains an aptamer domain, which can bind the metabolite, adenine, and a downstream expression platform, which contains the Shine-Dalgarno sequence (SD). When adenine is bound to the aptamer domain, the SD binds to the ribosomal unit for translation initiation denoted by $\mathrm{ON}$. When the riboswitch does not bind adenine, the SD forms a hairpin with the downstream expression platform (denoted by OFF), which prevents the ribosomal unit from recognizing the $\mathrm{SD}$, thus resulting in translation being inhibited. (b) Tertiary structure of the aptamer domain of the add A-riboswitch with adenine (shown in orange) bound. The aptamer forms a three-way junction composed of helix P1 and hairpins P2 and P3. Adenine binding stabilizes the tertiary loop-loop interactions between P2 and P3.

are involved in the control of both transcription and translation. Transcription termination occurs when the downstream expression platform forms a hairpin, or a terminator stem, followed by multi-U sequence, which results in weak interactions in the RNA-DNA hybrid, leading to disengagement of the polymerase from the DNA template. For riboswitches controlling translation initiation, the downstream hairpin stem typically contains the Shine-Dalgarno sequence, the binding site of the ribosomal unit (Figure $5 \mathrm{~b}$ ). Formation of the downstream hairpin, which involves a switch in the conformation of the aptamer domain (compare "ON" and "OFF" schematics in Figure 5a), inhibits the binding of a ribosomal unit needed for translation initiation.

Formation or disruption of the downstream stem, which depends on whether metabolite is bound or not, serves as a switch that controls gene expression. For riboswitches controlling transcription termination, the time needed for metabolite binding is typically on the order of seconds. For example, in purine-sensing riboswitches, ${ }^{50}$ one of the smallest riboswitches, there are 40-80 nucleotides between the aptamer domain and the multi-U sequence. With a typical transcription speed of 25-40 nucleotides per second, the time window is only $1-3 \mathrm{~s}$ for the metabolite to bind and stabilize the folded aptamer domain in order to influence transcription. On this time scale, riboswitches, with slow metabolite binding rates, would not reach thermodynamic equilibrium before the terminator sequence is transcribed. Hence, in this case, gene expression is under kinetic control. 
For riboswitches controlling translation initiation, folding influenced by metabolite binding has to compete with the binding of the ribosomal unit to the expression platform (Figure 5a). The rate of switching between different folding patterns must be faster than the binding time of the ribosomal unit for the riboswitch to function. In such riboswitches, gene expression could be under thermodynamic control since the translation initiation can occur after transcription is completed. The stability of the aptamer domain of riboswitches may have evolved to accommodate diverse demands imposed by distinct aspects of gene expression.

Two different but structurally identical adenine-sensing riboswitches (Figure 5b), pbuE and add adenine (A) riboswitches, that have been studied using pulling experiments $^{51}$ and simulations ${ }^{41}$ show similar aptamer structure but control gene expression through different functions. ${ }^{52-54}$ Both pbuE and add A-riboswitches are on-switch riboswitches, but pbuE A-riboswitch controls transcription termination while add A-riboswitch controls translation initiation. In order to describe the functions of riboswitches, it is important to understand the time scales associated with the folding of the aptamer, and the conformational changes involving part of the aptamer that forms the terminator hairpin by base pairing with the transcript in the downstream expression platform. In other words, one needs to understand in quantitative terms the folding landscape of the riboswitches in the presence and absence of metabolites so that the transition rates to specific states can be obtained. Such detailed information is best obtained using singlemolecule methods complemented by suitable theory.

Stability of Helices Determine f-Induced Unfolding of Purine Riboswitches. The adenine riboswitch aptamer is a three-way junction formed from helix P1 and hairpins P2 and P3 (Figure $5 b)$. The junction contains the binding pocket for adenine (Figure $5 \mathrm{~b}$ ), which is stabilized by tertiary interactions in the folded state. There is a kissing loop-loop interaction between helices P2 and P3 (Figure 5b), which is transient in the absence of metabolites, and is stabilized only when the aptamer binds the ligand.

A single-molecule experiment first observed the hierarchical folding in the $p b u E$ adenine riboswitch aptamers ${ }^{51}$ by measuring the extension of the riboswitch aptamer as a function of $f$. In the absence of the metabolite, two clear steps in the change of contour lengths were found, which were associated with the unfolding of helices P2 and P3. Because of the difference in the lengths of the helices P2 and P3 (19 and 21 nucleotides, respectively), the order of unfolding of the two helices could be unambiguously assigned. The measured folding landscape shows (Figure 6a) that helix P3 is ruptured before P2 in the pbuE A-riboswitch aptamer. With metabolite bound, larger changes in contour lengths with larger forces were found, and unfolding occurred in a single step. This is due to the stabilization of the folded aptamer structure by the bound metabolite, which makes unfolding of helix P1 and the binding pocket the major free energy barrier in the unfolding process. Figure 6a shows that refolding from an initially unfolded state occurs with the dominant pathway being order of $P 2 \rightarrow P 3 \rightarrow$ $P 1$.

A theoretical study of the add adenine riboswitch aptamer using a coarse-grained self-organized polymer model also found multiple intermediate steps in the folding landscape of the riboswitch aptamer. ${ }^{41}$ In order to ascertain the folding mechanism, simulations were performed by quenching the force from an initial high force to constant low forces. During
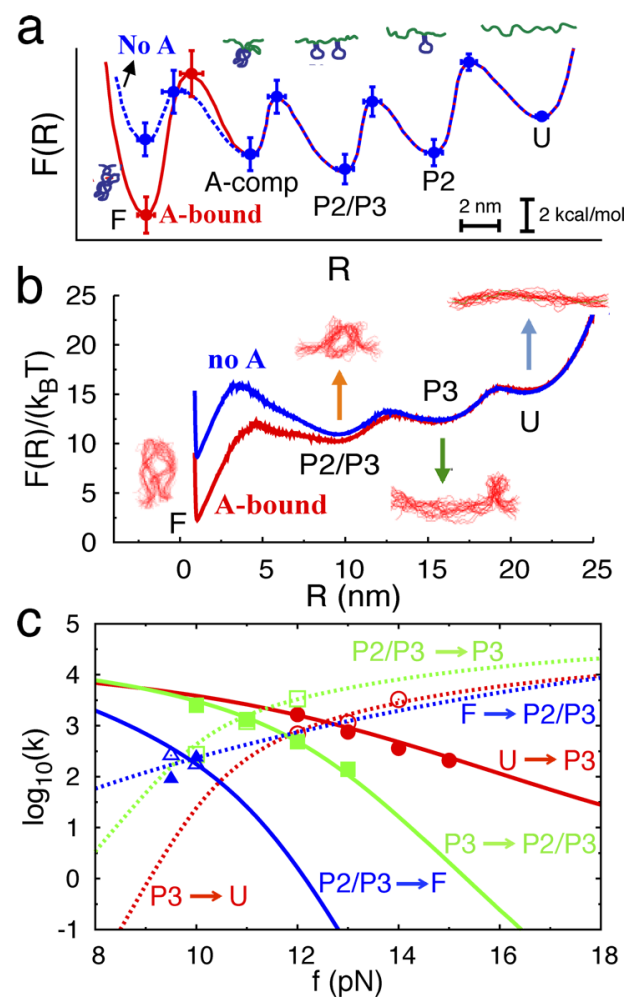

Figure 6. (a) Free energy profile extracted and reconstructed from LOT experiments for $p b u E$ A-riboswitch with and without adenine being bound at $f=6.5 \mathrm{pN}^{17}$ The structural elements (see Figure $5 \mathrm{~b}$ ) that are intact in each state are indicated. (b) Folding landscape for add A-riboswitch aptamer as a function of extension $\mathrm{R}$ at $f=10 \mathrm{pN}$ without and with adenine. The ensemble of structures in the intermediate states are shown. (c) The logarithm of the transition rates, $\log (k=1 / \bar{\tau})$, between the distinct states calculated using the theory of mean first passage times (eq 1, shown in lines), and directly from the time traces of the extension of a coarse-grained model of the aptamer generated using Brownian dynamics simulations (shown as points).

the folding process P3 forms first followed by P2, and finally the triple-helix junction and the helix P1 form. The order of folding can be directly associated with the stability of individual helices. ${ }^{41}$ Indeed, the isolated P3 is more stable than P2 by about $1 \mathrm{kcal} / \mathrm{mol}$, as predicted by the Vienna RNA package, ${ }^{55}$ thus explaining its early formation.

\section{An organization principle that emerges from simulations and theory is that the stability of the individual helices determines the order of assembly of riboswitches and pseudoknots.}

Remarkably, despite the structural similarity between $p b u E$ and add A-riboswitch aptamers, experiments show that P2 in pbuE unfolds last (Figure 6a) and presumably is the first structural element to refold. The organization principle that emerges is that assembly of RNA is largely determined by the stability of individual helices, which implies that in the pbuE Ariboswitch aptamer, P2 ought to be more stable than P3. Indeed, the relative stability of $\mathrm{P} 2 / \mathrm{P} 3$ is different in the $p b u E \mathrm{~A}$ riboswitch aptamer. The predicted free energy for the 
secondary structure of $\mathrm{P} 2$ is smaller than that of $\mathrm{P} 3$ by $2 \mathrm{kcal} /$ mol. The stability difference explains the reversed order of the folding of P2 and P3 shown in the folding landscapes. An organization principle that emerges from simulations and theory is that the stability of the individual helices determines the order of assembly of riboswitches and pseudoknots.

Our prediction for the folding landscape of add adenine riboswitch (Figure 6b) was quantitatively validated in a recent single-molecule experiment on add A-riboswitch. ${ }^{56}$ The experiments showed that helix $\mathrm{P} 1$ in add A-riboswitches is more stable than that in $p b u E$ A-riboswitches. In $p b u E$ Ariboswitches, helix P1 unfolds at forces as low as $\sim 3 \mathrm{pN}$ without metabolite being bound. ${ }^{51}$ In add A-riboswitches, forces $\sim 10 \mathrm{pN}$ are needed to unfold helix P1. ${ }^{41,56}$ The folding landscapes of these two purine riboswitches, obtained from experiments and simulations, show that the assembly of riboswitches is determined by the local stability of the structural elements in these two purine riboswitch aptamers. The principle linking folding mechanism to the stability of individual helices is general and has been further illustrated using folding of a number of pseudoknots ${ }^{57}$ whose folding pathways could be accurately predicted using the stability of the individual helices. It might appear that entropic effects could become relevant when folding takes place spontaneously (in the absence of force), thus invalidating the proposed organization principle. This is not the case as simulations have explicitly demonstrated. ${ }^{57}$ More importantly, the organization principle described here has been used to quantitatively rationalize temperature jump experiments on RNA pseudoknots. ${ }^{13}$

Transition Rates between the Network of Connected States from add A-Riboswitch. Just as for RNA hairpins, the matrix of transition rates connecting the various states $(\mathrm{F}, \mathrm{P} 2 / \mathrm{P} 3, \mathrm{P} 3$, and $\mathrm{U}$ in Figure $6 \mathrm{c}$ ) could be calculated using the free energy profile in Figure $6 \mathrm{~b}$. The $f$-dependent transition rate between any two states can be obtained from the time traces of the extension, which would require generating a large number of folding trajectories at each $f$. Alternatively, $F(f, R)$ calculated from accurate estimates at one force could be used to obtain the rates at other forces based upon the theory of mean first passage times (eq 1). Following the procedure used for obtaining hopping rates for hairpins away from $f_{\mathrm{m}}{ }^{28}$ we calculated the diffusion coefficients (needed to obtain hopping rates between multiple states of the riboswitch) by equating the transition rate calculated using Kramers theory to that obtained from time traces generated using simulations at a specified $f$. These effective diffusion coefficients reflect collective processes associated with global folding of the riboswitch. Thus, we expect that, generically, $D \approx \sigma^{2} / \tau_{0}$, where $\tau_{0}\left(\approx 10^{-6} \mathrm{~s}\right)$ is the prefactor in RNA folding. ${ }^{6,58}$ If $\sigma=0.7 \mathrm{~nm}$, then the calculated value of $D \approx 10^{4} \mathrm{~nm}^{2} / \mathrm{s}$, which is in reasonable agreement with the numerical values needed for obtaining agreement between rates from simulations and from free energy profiles. ${ }^{41}$ Using the values of $D$, the hopping rates between the various states in the add A-riboswitch were calculated over a range of forces using eq 1 with $F(R, f)$ at one value of $f$ ! Comparison of rates obtained using $F(R, f)$ and eq 1 and numerically exact results obtained from simulations (Figure 6c) is excellent. This might suggest that $R$ may be a reasonable reaction coordinate for describing riboswitch folding. However, when we assess whether the location of the barrier height describes the actual transition state ensemble for the $F \rightarrow P 2 / P 3$ transition using the molecular tensegrity parameter, it is found that $P_{\text {fold }}$ deviates substantially from 0.5 . Thus, it is unlikely that for complex structures such as riboswitches, and more generally ribozymes, the pulling coordinate alone can describe the folding process.

Although fundamentally new insights into the folding of RNA have emerged from subjecting them to tension, there are many major unresolved questions, which will require a combination of theory, simulations and experiments. Here are a few of them. (i) In the most favorable circumstances the folding landscape and hopping rates can only be obtained at force values at which RNA makes multiple transitions. Can these estimates be used to extrapolate to zero force? (ii) Applications here demonstrate that extension is not always a good reaction coordinate. If this is the case, how can one profitably utilize the high quality folding trajectories to map the network of connected states, which could be hidden in $R$ but become transparent in other auxiliary variables? (iii) Even if $R$ is a good reaction coordinate, are the extracted values for mean barrier heights at zero force are reliable? This issue is exacerbated because, in general, one expects a distribution of barrier heights for RNA and proteins, ${ }^{59}$ and hence the mean value may not be informative. In addition, independent measurements of the absolute values of barrier heights are difficult, thus making it hard to assess the accuracy of estimates based on single-molecule folding trajectories. Only by resolving these issues can the full scope and power of single-molecule pulling experiments in biology be realized.

\section{AUTHOR INFORMATION}

Notes

The authors declare no competing financial interest.

\section{Biographies}

Jong-Chin Lin received his Ph.D. in Physics at the University of California at Davis, under the direction of Prof. Daniel L. Cox. He is currently working as a postdoctoral fellow at the University of Maryland at College Park. He is interested in applying quantitative methods to study problems in biology.

Changbong Hyeon is a Professor at the Korea Institute for Advanced Study. His research interests include self-assembly and mechanical response of biopolymers, theory for single-molecule measurements, protein allostery, polymer physics, and systems biology. http:// newton.kias.re.kr/ hyeoncb

D. Thirumalai is a Distinguished University Professor at the University of Maryland. His research interests are in soft matter science, biophysics, and condensed matter science. http://biotheory. umd.edu

\section{ACKNOWLEDGMENTS}

We thank the National Science Foundation (CHE 09-4033) and the National Institutes of Health (GM089685) for supporting this work.

\section{REFERENCES}

(1) Doudna, J.; Cech, T. The Chemical Repertoire of Natural Ribozymes. Nature 2002, 418, 222-228.

(2) Nissen, P.; Hansen, J.; Ban, N.; Moore, P.; Steitz, T. The Structural Basis of Ribosome Activity in Peptide Bond Synthesis. Science 2000, 289, 920-930.

(3) Matke, M. A; Birchier, J. A. RNAi-Mediated Pathways in the Nucleus. Nat. Rev. Genet. 2005, 6, 24-35.

(4) Winkler, W. C; Breaker, R. R. Regulation of Bacterial Gene Expression by Riboswitches. Annu. Rev. Microbiol. 2005, 59, 487-517.

(5) Montange, R. K; Batey, R. Riboswitches: Emerging Themes in RNA Structure and Function. Annu. Rev Biophys. 2008, 37, 117-133. 
(6) Thirumalai, D.; Hyeon, C. RNA and Protein folding: Common Themes and Variations. Biochemistry 2005, 44, 4957-4970.

(7) Dima, R. I; Hyeon, C.; Thirumalai, D. Extracting Stacking Interaction Parameters for RNA from the Data Set of Native Structures. J. Mol. Biol. 2005, 347, 53-69.

(8) Heilman-Miller, S.; Pan, J.; Thirumalai, D.; Woodson, S. Role of Counterion Condensation in Folding of the Tetrahymena Ribozyme II. Counterion-Dependence of Folding Kinetics. J. Mol. Biol. 2001, 309, 57-68.

(9) Guo, Z.; Thirumalai, D.; Honeycutt, J. D. Folding Kinetics of Proteins: A Model Study. J. Chem. Phys. 1992, 97, 525-535.

(10) Guo, Z.; Thirumalai, D. Kinetics of Protein Folding: Nucleation Mechanism, Time Scales, and Pathways. Biopolymers 1995, 36, 83102.

(11) Pan, J.; Thirumalai, D.; Woodson, S. A. Folding of RNA Involves Parallel Pathways. J. Mol. Biol. 1997, 273, 7-13.

(12) Zhuang, X.; Bartley, L.; Babcock, A.; Russell, R.; Ha, T.; Hershlag, D.; Chu, S. A Single-Molecule Study of RNA Catalysis and Folding. Science 2000, 288, 2048-2051.

(13) Narayanan, R.; Velmurugu, Y.; Kuznetsov, S.; Ansari, A. Fast Folding of RNA Pseudoknots Initiated by Laser Temperature-Jump. J. Am. Chem. Soc. 2011, 133, 18767-18774.

(14) Woodside, M. T; Garcia-Garcia, C.; Block, S. M. Folding and Unfolding Single RNA Molecules under Tension. Curr. Opin. Chem. Biol. 2008, 12, 640-646.

(15) Liphardt, J.; Onoa, B.; Smith, S. B; Tinoco, I., Jr.; Bustamante, C. Reversible Unfolding of Single RNA Molecules by Mechanical Force. Science 2001, 292, 733-737.

(16) Onoa, B.; Dumont, S.; Liphardt, J.; Smith, S. B; Tinoco, I., Jr.; Bustamante, C. Identifying Kinetic Barriers to Mechanical Unfolding of the T. thermophila Ribozyme. Science 2003, 299, 1892-1895.

(17) Woodside, M. T; Anthony, P. C; Behnke-Parks, W. M; Larizadeh, K.; Herschlag, D.; Block, S. M. Direct Measurement of the Full, Sequence-Dependent Folding Landscape of a Nucleic Acid. Science 2006, 314, 1001-1004.

(18) Hyeon, C.; Thirumalai, D. Capturing the Essence of Folding and Functions of Biomolecules Using Coarse-Grained Models. Nat. Commun. 2011, 2, 487.

(19) Sim, A. Y. L; Minary, P.; Levitt, M. Modeling Nucleic Acids. Curr. Opin. Struct. Biol. 2012, 22, 273-278.

(20) Cole, P. E; Yang, S. K; Crothers, D. M. Conformational Changes of Transfer Ribonucleic Acid. Equilibrium Phase Diagrams. Biochemistry 1972, 11, 4358-4368.

(21) Pörschke, D.; Uhlenbeck, O.; Martin, F. Thermodynamics and Kinetics of the Helix-Coil Transition of Oligomers Containing GC Base Pairs. Biopolymers 1973, 12, 1313-1335.

(22) Porschke, D. Thermodynamic and Kinetic Parameters of an Oligonucleotide Hairpin Helix. Biophys. Chem. 1974, 1, 381-386.

(23) Crothers, D.; Cole, P.; Hilbers, C.; Shulman, R. The Molecular Mechanism of Thermal Unfolding of Escherichia coli Formylmethionine Transfer RNA. J. Mol. Biol. 1974, 62, 383-401.

(24) Bloomfield, V. A.; Crothers, D. M.; Tinico, I., Jr. Nucleic Acids, Structures, Properties and Functions; University Science Books: Sausalito, CA, 2000

(25) Ma, H.; Proctor, D. J; Kierzek, E.; Kierzek, R.; Bevilacqua, P. C; Gruebele, M. Exploring the Energy Landscape of a Small RNA Hairpin. J. Am. Chem. Soc. 2006, 128, 1523-1530.

(26) Ma, H.; Wan, C.; Wu, A.; Zewail, A. DNA Folding and Melting Observed in Real Time Redefine the Energy Landscape. Proc. Natl. Acad. Sci. U.S.A. 2007, 104, 712-716.

(27) Woodside, M. T; Behnke-Parks, W. M; Larizadeh, K.; Travers, K.; Herschlag, D.; Block, S. M. Nanomechanical Measurements of the Sequence-Dependent Folding Landscapes of Single Nucleic Acid Hairpins. Proc. Natl. Acad. Sci. U.S.A. 2006, 103, 6190-6195.

(28) Hyeon, C.; Morrison, G.; Thirumalai, D. Force Dependent Hopping Rates of RNA Hairpins Can Be Estimated from Accurate Measurement of the Folding Landscapes. Proc. Natl. Acad. Sci. U.S.A. 2008, 105, 9604-9606.
(29) Hyeon, C.; Thirumalai, D. Mechanical Unfolding of RNA Hairpins. Proc. Natl. Acad. Sci. U.S.A. 2005, 102, 6789-6794.

(30) Hyeon, C.; Thirumalai, D. Multiple Probes Are Required to Explore and Control the Rugged Energy Landscape of RNA Hairpins. J. Am. Chem. Soc. 2008, 130, 1538-1539.

(31) de Messieres, M.; Brawn-Cinani, B.; Porta, A. L. Measuring the Folding Landscape of a Harmonically Constrained Biopolymer. Biophys. J. 2011, 100, 2736-2744.

(32) Hyeon, C.; Dima, R. I; Thirumalai, D. Pathways and Kinetic Barriers in Mechanical Unfolding and Refolding of RNA and Proteins. Structure 2006, 14, 1633-1645.

(33) Garcia, A. E; Paschek, D. Simulation of the Pressure and Temperature Folding/Unfolding Equilibrium of a Small RNA Hairpin. J. Am. Chem. Soc. 2008, 130, 815-817.

(34) Bowman, G. R; Huang, X.; Yao, Y.; Sun, J.; Carlsson, G.; Guibas, L. J; Pande, V. S. Structural Insight into RNA Hairpin Folding Intermediates. J. Am. Chem. Soc. 2008, 130, 9676-9678.

(35) Hyeon, C.; Thirumalai, D. Forced-Unfolding and Force-Quench Refolding of RNA Hairpins. Biophys. J. 2006, 90, 3410-3427.

(36) Hinczewski, M.; Thirumalai, D. Proc. Natl. Acad. Sci. USA. 2012, in press.

(37) Hyeon, C.; Thirumalai, D. Mechanical Unfolding of RNA: From Hairpins to Structures with Internal Multiloops. Biophys. J. 2007, 92, 731-743.

(38) Li, P. T. X; Collin, D.; Smith, S. B; Bustamante, C.; Tinoco, I., Jr. Probing the Mechanical Folding Kinetics of TAR RNA by Hopping, Force-Jump, and Force-Ramp Methods. Biophys. J. 2006, 90, 250-260.

(39) Li, P. T. X; Bustamante, C.; Tinoco, I., Jr. Real-Time Control of the Energy Landscape by Force Directs the Folding of RNA Molecules. Proc. Natl. Acad. Sci. U.S.A. 2007, 104, 7039-7044.

(40) Dudko, O. K; Hummer, G.; Szabo, A. Intrinsic Rates and Activation Free Energies from Single-Molecule Pulling Experiments. Phys. Rev. Lett. 2006, 96, 108101.

(41) Lin, J.; Thirumalai, D. Relative Stability of Helices Determines the Folding Landscape of Adenine Riboswitch Aptamers. J. Am. Chem. Soc. 2008, 130, 14080-14081.

(42) Zhurkov, S. N. Kinetic Concept of the Strength of Solids. Int. J. Fract. Mech. 1965, 1, 311-322.

(43) Bell, G. I. Models for the Specific Adhesion of Cells to Cells. Science 1978, 200, 618-627.

(44) Klosek, M. M; Matkowsky, B. J; Schuss, Z. Kramers Problem in the Turnover Regime - The Role of the Stochastic Separatrix. Ber. Bunsenges. Phys. Chem. 1991, 95, 331.

(45) Morrison, G.; Hyeon, C.; Hinczewski, M.; Thirumalai, D. Compaction and Tensile Forces Determine the Accuracy of Folding Landscape Parameters from Single Molecule Pulling Experiments. Phys. Rev. Lett. 2011, 106, 138102.

(46) Ingber, D. E.; Tensegrity, I. Cell Structure and Hierarchical Systems Biology. J. Cell. Sci. 2003, 116, 1157-1173.

(47) Edwards, S. A; Wagner, J.; Gräter, F. Dynamic Prestress in a Globular Protein. PLoS Comput. Biol. 2012, 8, e1002509.

(48) Fuller, B. Tensegrity. Portfolio Artnews Annu. 1961, 4, 112-127. (49) Sudarsan, N.; Lee, E. R; Weinberg, Z.; Moy, R. H; Kim, J. N; Link, K. H; Breaker, R. R. Riboswitches in Eubacteria Sense the Second Messenger Cyclic Di-GMP. Science 2008, 321, 411-413.

(50) Edwards, T. E; Klein, D. J; Ferre-DAmare, A. R. Riboswitches: Small Molecule Recognition by Gene Regulatory RNAs. Curr. Opin. Struct. Biol. 2004, 11, 1729-1741.

(51) Greenleaf, W. J; Frieda, K. L; Foster, D. A. N; Woodside, M. T; Block, S. M. Direct Observation of Hierarchical Folding in Single Riboswitch Aptamers. Science 2008, 319, 630-633.

(52) Serganov, A.; Yuan, Y. T; Pikovskaya, O.; Polonskaia, A.; Malinina, L.; Phan, A. T; Hobartner, C.; Micura, R.; Breaker, R. R; Patel, D. Structural Basis for Discriminative Regulation of Gene Expression by Adenine- and Guanine-Sensing mRNAs. J. Chem. Biol. 2004, 11, 1729-1741.

(53) Mandal, M.; Boese, B.; Barrick, J. E; Winkler, W. C; Breaker, R R. Riboswitches Control Fundamental Biochemical Pathways in Bacillus subtilis and Other Bacteria. Cell 2003, 113, 577-586. 
(54) Mandal, M.; Breaker, R. R. Adenine Riboswitches and Gene Activation by Disruption of a Transcription Terminator. Nat. Struct. Mol. Biol. 2004, 11, 29-35.

(55) Hofacker, I. Vienna RNA Secondary Structure Server. Nucleic Acids Res. 2003, 31, 3429.

(56) Neupane, K.; Daniel, H. Y; Foster, A. N; Wang, F.; Woodside, M. T. Single-Molecule Force Spectroscopy of the Add Adenine Riboswitch Relates Folding to Regulatory Mechanism. Nucleic Acids Res. 2011, 39, 7677-7687.

(57) Cho, S.; Pincus, D.; Thirumalai, D. Assembly Mechanisms of RNA Pseudoknots Are Determined by the Stabilities of Constituent Secondary Structures. Proc. Natl. Acad. Sci. U.S.A. 2009, 106, 17349.

(58) Hyeon, C.; Thirumalai, D. Chain Length Determines the Folding Rates of RNA. Biophys. J. 2012, 102, L11-L13.

(59) Klimov, D. K; Thirumalai, D. Lattice Model Studies of ForceInduced Unfolding of Protein. J. Phys. Chem. B 2001, 105, 6648-6654. 